\title{
A Yellow Polariton Condensate in a Dye filled Microcavity
}

Tamsin Cookson $^{1+}$, Kyriacos Georgiou ${ }^{2+}$, Anton Zasedatelev ${ }^{3}$, Richard T. Grant ${ }^{2}$, Tersilla Virgili ${ }^{4}$, Marco Cavazzini ${ }^{4}$, Francesco Galeotti ${ }^{4}$, Caspar Clark $^{5}$, Natalia G. Berloff ${ }^{3,6}$, David G. Lidzey ${ }^{2 *}$ and Pavlos G. Lagoudakis ${ }^{1,3^{*}}$

1. Department of Physics and Astronomy, The University of Southampton, University Road, Southampton SO17 1BJ, U.K.

2. Department of Physics and Astronomy, The University of Sheffield, Hicks Building, Hounsfield Road, Sheffield S3 7RH, U.K.

3. Skolkovo Institute of Science and Technology, Moscow 143026, Russia.

4. IFN, ISMAC and ISTM - CNR Milano, Italy.

5. Helia Photonics Ltd, 2 Rosebank Rd, Livingston EH54 7EJ, U.K.

6. Department of Applied Mathematics and Theoretical Physics, University of Cambridge, Cambridge, CB3 0WA.

*Authors for correspondence: d.g.lidzey@sheffield.ac.uk; pavlos.lagoudakis@soton.ac.uk

${ }^{+}$T.C. and K.G. contributed equally to this work. 
Abstract: We observe polariton condensation in the yellow part of the visible spectrum from a planar organic semiconductor microcavity containing the molecular dye BODIPY-Br. We provide experimental fingerprint of polariton condensation under non-resonant optical excitation, including the non-linear dependence of the emission intensity and wavelength blueshift with increasing excitation density, single excitation pulse dispersion imaging and real space interferometry. The latter two allow us to visualise the collapse of the energy distribution and the long-range coherence of the polariton condensate. 
Bose-Einstein condensation (BEC) is an exotic state of matter, wherein particles coalesce to a macroscopically occupied coherent state. BECs have been observed for a broad range of systems such as ${ }^{4} \mathrm{He},{ }^{[1]}$ alkali-metal atoms, ${ }^{[2]}$ magnons, ${ }^{[3]}$ and polaritons. ${ }^{[4]}$ Beyond the beauty of the underlying physics describing the fundamental properties and dynamics of BECs, ${ }^{[5]}$ there is a range of applications that utilise the coherence of their massive wavefunctions, especially in the rapidly developing field of quantum technologies. ${ }^{[6]}$ Unlike other BECs, polariton condensates (hosted in semiconductor slabs embedded in optical cavities) can optically be pumped, and more importantly are interrogated through photoluminescence. Indeed, due to finite cavity lifetimes, polaritons decay in the form of photons that carry all information of the corresponding polariton state (energy, momentum, spin and phase). By appropriate choice of the crystalline semiconductor host, polariton condensates can form even at room temperature. ${ }^{[7,8]}$ Circumventing the challenges of epitaxial growth, polariton condensates were also realised in soft-matter organic microcavities. ${ }^{[9,10]}$ The room temperature operation and ease with which organic polariton condensates can be "written" and "read" is attractive for imprinting polariton lattices, a promising platform for quantum simulators. ${ }^{[11]}$

A number of very different molecular systems have been used to date as the semiconductor host in optical cavities to demonstrate polariton condensation, varying from crystalline anthracene to oligofluorenes, conjugated polymers and fluorescent proteins. ${ }^{[12,9,10,13]}$ Although the chemical structure and morphology of these molecular hosts are very dissimilar, all three have relatively narrow absorption spectra and small Stokes shift with respect to their optical oscillator strengths. These features are necessary for strong coupling, but not sufficient for polariton condensation. For example, J-aggregates have very similar spectroscopic characteristics with the above molecular systems allowing for strong coupling, however, in J-aggregates polariton condensates remains elusive even under resonant excitation schemes. ${ }^{[14]}$ A characteristic of the molecular systems that have exhibited polariton condensation is their relatively high quantum yield (QY), >10\%, even with 
increasing excitation densities. It is plausible that a key ingredient for polariton condensation in molecular systems is the balance between radiative and non-radiative decay paths, since the latter will limit the number of excitons available to form polaritons. Undoubtedly, there is still a plethora of molecular systems that satisfy the above conditions and other considerations should be taken into account to address limitation beyond the formation of a polariton condensate. For any application that utilises the macroscopic wavefunction of organic polariton condensates to become viable, control over the disorder and its adverse effects on spatial coherence and homogeneity of the polariton energy landscape need to be addressed. Here, we demonstrate evidence of polariton condensation in a different class of organic semiconductors, that of a molecular dye diluted in a matrix polymer, resulting in extended polariton condensates.

The material investigated is a bromine-substituted boron-dipyrromethene (BODIPY-Br) that combines relatively high photoluminescence quantum yield, between $10 \%$ and $50 \%$ (dependent on sample preparation conditons), with fluorescence emission around $550 \mathrm{~nm} .{ }^{[15]}$ To incorporate BODIPY-Br into a microcavity, it is necessary to disperse it into a transparent polymeric matrix at relatively low concentration to limit unwanted molecular aggregation and luminescence quenching phenomena. ${ }^{[16-18]}$ This approach to creation of a medium is different from previous studies in which a pure film of organic chromophores was used. ${ }^{[9,12]}$

Figure 1(a) shows the chemical structure of the molecular dye BODIPY-Br, together with its absorption and fluorescence when dispersed into a transparent polystyrene matrix at a concentration of $10 \%$ by mass. It can be seen that the absorption maximum of BODIPY-Br peaks at $530 \mathrm{~nm}$, whilst its fluorescence maximum is red-shifted to $550 \mathrm{~nm}$. We have previously explored the photophysics of microcavities containing BODIPY-Br, and have concluded that weakly-coupled excimer-like states, together with emission from the $(0,1)$ vibrational transition that are both located 
around $593 \mathrm{~nm}$ are responsible for optically pumping polariton states along the lower polariton branch. $^{[15]}$

To explore the non-linear emission properties of BODIPY-Br, we used a stripe-pumping geometry to generate amplified spontaneous emission from a non-cavity control film. Here a $186 \mathrm{~nm}$ thick non-cavity film of BODIPY-Br in polystyrene ( $10 \%$ by mass) was spin-cast onto a quartz substrate and then pumped using $100 \mathrm{fs}$ laser pulses at a repetition rate of $1 \mathrm{kHz}$ at $500 \mathrm{~nm}$ along a $20 \mathrm{~mm}$ long stripe. Figure 1(b) shows emission from the film at various pump fluences. Here, it can be seen that at threshold of $104.1 \mu \mathrm{J} / \mathrm{cm}^{2}$, there is a strong increase in emission at around $589 \mathrm{~nm}$ with the emergence of a peak having a linewidth of around $\sim 8 \mathrm{~nm}(28 \mathrm{meV})$. This wavelength approximately coincides with the $(0,1)$ vibrational transition, suggestive of a 4-level lasing system. This measurement usefully indicates that BODIPY-Br can support optical amplification, which is indicative of low optical losses under high excitation density, rendering it a promising candidate for realising polariton condensation.

We fabricate BODIPY-Br into a microcavity by spin-casting a $186 \mathrm{~nm}$ thin film onto a distributed Bragg reflector (DBR) consisting of 10 pairs of $\mathrm{SiO}_{2} / \mathrm{Nb}_{2} \mathrm{O}_{5}$. We deposit a second 8-pair $\mathrm{SiO}_{2} /$ $\mathrm{Nb}_{2} \mathrm{O}_{5}$ DBR onto the BODIPY-Br film using ion assisted electron beam and reactive sublimation, as shown in the schematic of the cavity structure in Figure 2(a). The measured Q-factor of the resulting cavity is $\sim 440$ corresponding to a cavity lifetime of $133 \mathrm{fs}$. Figure 2(b) shows white light reflectivity spectra as a function of the off-axis viewing angle, where the measurements are made through the 8-pair DBR. It can be seen that two optical modes are clearly visible undergoing anticrossing around a wavelength of $530 \mathrm{~nm}$; a wavelength that we associate with the $(0,0)$ monomer absorption transition of the BODIPY-Br. Figure 2(c) shows the energy of the upper and lower polariton branches determined from the reflectivity measurements. The data are fitted to a standard two-level oscillator model from which we obtain a Rabi-splitting energy of $91 \mathrm{meV}$. The energy of 
the exciton energy of the fit $(2.33 \mathrm{eV})$ closely coincides with the peak absorption energy of the BODIPY-Br $(0,0)$ electronic transition $(2.34 \mathrm{eV})$. We overlay the dispersion plot with an intensity map of the photoluminescence emitted by the cavity under non-resonant pumping. Here, we excite our sample using a bandpass filter centred at $450 \mathrm{~nm}$ with $10 \mathrm{~nm}$ bandwidth to spectrally filter a 6 ps pulsed super-continuum laser operating at $40 \mathrm{MHz}$. The excitation density for these measurements is $1.2 \mu \mathrm{J} / \mathrm{cm}^{2}$. It can be seen that the cavity emission is most intense around the bottom of the lower polariton branch (corresponding to $\mathrm{k}_{/ /}=0$ ), and reduces in intensity towards the energy of the uncoupled exciton. We have previously shown that the distribution of emission along the lower polariton branch (LPB) is primarily determined by the distribution of weakly-coupled states within the exciton reservoir. ${ }^{[15]}$ Figure 2(d) shows the free space yellow emission from our cavity under non-resonant pumping.

We investigate the non-linear photoluminescence dynamics using 2 ps optical pulses at $400 \mathrm{~nm}$. The sample is held in a vacuum chamber at $10^{-6} \mathrm{mbar}$ at room temperature. The full width at half maximum of the pump beam on the sample is $\sim 8 \mu \mathrm{m}$. Photoluminescence is collected using a lens with 0.42 numerical aperture that allows for dispersion and real space imaging using an electron multiplication charge coupled device (CCD) camera at the exit slit of a $55 \mathrm{~cm}$ spectrophotometer and a grating of 1200 grooves/millimetre. We have synchronised the timing of the optical detection system with the train of excitation pulses down to a single pulse excitation/image acquisition. Using the setup described above we can perform single pulse excitation dispersion imaging. Also with the use of a stabilised Michelson interferometer, we can record interferograms that allow us to measure the extent of the coherence of the emissive states under single pulse excitation.

Figures 3(a) - (c) show dispersion images recorded below, near and twice above threshold. Figure 3(a) shows the linear dispersion time averaged over 1500 excitation pulses. With increasing pump intensity, at a threshold excitation density we observe the collapse of the emission to the bottom of 
the polariton dispersion, centred on $\mathrm{k}_{/ /}=0$, as shown in the dispersion image of Fig. $3(\mathrm{~b})$ that is time averaged over 2 excitation pulses. Figure 3(c) shows the dispersion image at approximately twice above threshold, integrated over 4 excitation pulses, where the emission appears blueshifted with respect to the linear regime. In Figure 3(d), we plot the photoluminescence spectra extracted at $\mathrm{k}_{/ /}=$ 0 from dispersion images recorded for different pump intensities and normalised to the number of excitation pulses used per recording. Evidently, as the pump intensity increases the spectrum gets narrower and blueshifts with respect to the spectrum at the lowest excitation density (black curve in Fig. 3(d)). In Figure 3(e), we plot the integrated intensity of the above spectra and the corresponding linewidth measured at FWHM as a function of the upper bound of the threshold excitation density $\left(527.3 \mu \mathrm{J} / \mathrm{cm}^{2}\right)$. We observe a threshold-like behaviour at $527.3 \mu \mathrm{J} / \mathrm{cm}^{2}$ accompanied by a reduction in linewidth from 2.8 to $0.5 \mathrm{~nm}$. Figure 3(f) shows a continuous blueshift of the photoluminescence spectrum by up to $5 \mathrm{meV}$, occurring at twice the threshold density of that observed by Plumhof et al. ${ }^{[10]}$ who studied a cavity containing a conjugated polymer. In areas on the sample where we do not observe polariton condensation, there is no visible blue-shift of the dispersion. For the detuning used here, we calculate the exciton content, $\left|\mathrm{X}_{\mathrm{LP}}\right|^{2}$, to be 0.103 at $\mathrm{k}_{/ /}=0$ of the lower polariton branch. We note here that by ramping the excitation density twice above threshold and back to the linear regime the optical properties of the sample remain virtually unchanged, including the shape of the photoluminescence spectrum. The observed non-linearity on the excitation density associated with the continuous blue-shift of the photoluminescence spectrum provide strong evidence of polariton condensation.

Figure 4(a) shows the interferogram of a polariton condensate at threshold excitation density for a single excitation pulse utilising a stabilised Michelson interferometer equipped with a retro-reflector to generate optical interference between the emitted light by the cavity and its inverted image. This technique has been utilised to realise experimentally the long range phase coherence of the condensate in many previous organic condensate studies. ${ }^{[19-22]}$ The interference fringes across the 
image are plotted in Fig. 4(b) and are fitted with a convoluted Gaussian (red curve). The visibility contrast of $86 \%$ indicates a high degree of spatial coherence across the condensate that extends beyond the excitation spot. From the standard deviation of the Gaussian fit we calculate a coherence length of $28 \mu \mathrm{m}$ from $\lambda_{c}=2 \sqrt{2 \pi} \sigma$, suggesting a homogeneous polariton energy landscape of the same order.

In conclusion, we report on a yellow polariton condensate in a dye filled microcavity. We obtain strong evidence of non-linear photoluminescence with increasing excitation density, associated with a six-fold linewidth narrowing and a continuous blue-shift attributed to polariton interactions with other polaritons and the exciton reservoir. The wavelength of the polariton condensate is blueshifted with respect to the wavelength, where amplified spontaneous emission occurs in BODIPY$\mathrm{Br}$ (565 and $589 \mathrm{~nm}$ respectively) indicating that the non-linearity does not necessarily coincide with the point of maximum gain of the molecular dye. Furthermore, single shot interferometry reveals substantial long range coherence across the condensate and uniformity of the polariton energy landscape. The latter is important for expanding this work to lattices of polariton condensates and their applications in polariton simulators. It is conceivable that there is a large number of different molecular dyes that could be dispersed into a polymer matrix and embedded in a cavity that would allow for polariton condensation to be realised at wavelengths spanning the entire visible and near infrared. This wavelength selectivity could become of interest in the development of future optoelectronic devices, including hybrid organic-inorganic polariton laser diodes.

We thank EPSRC for the funding this research through the Programme Grant EP/M025330/1 "Hybrid Polaritonics" and for funding PhD scholarships for T.C., K.G. and R.T.G. through institutional DTP allocations. K.G. fabricated the sample and characterised the linear dynamics of 
the microcavity. T.C. contributed the non-linear spectroscopy and A.Z. contributed the ASE measurements.

\section{References}

[1] F. Pereira Dos Santos, J. Léonard, J. Wang, C. J. Barrelet, F. Perales, E. Rasel, C. S. Unnikrishnan, M. Leduc, C. Cohen-Tannoudji, Phys. Rev. Lett. 2001, 86, 3459.

[2] M. H. Anderson, J. R. Ensher, M. R. Matthews, C. E. Wieman, E. A. Cornell, Science 1995, 269, 198.

[3] V. Zapf, M. Jaime, C. D. Batista, Rev. Mod. Phys. 2014, 86, 563.

[4] J. Kasprzak, M. Richard, S. Kundermann, A. Baas, P. Jeambrun, J. M. J. Keeling, F. M. Marchetti, M. H. Szymańska, R. André, J. L. Staehli, V. Savona, P. B. Littlewood, B. Deveaud, L. S. Dang, Nature 2006, 443, 409.

[5] J. R. Anglin, W. Ketterle, Nature 2002, 416, 211.

[6] K. R. Patton, U. R. Fischer, Phys. Rev. A 2013, 87, 52303.

[7] G. Christmann, R. Butté, E. Feltin, J.-F. Carlin, N. Grandjean, Appl. Phys. Lett. 2008, 93, 51102.

[8] T.-C. Lu, Y.-Y. Lai, Y.-P. Lan, S.-W. Huang, J.-R. Chen, Y.-C. Wu, W.-F. Hsieh, H. Deng, Opt. Express 2012, 20, 5530.

[9] K. S. Daskalakis, S. a Maier, R. Murray, S. Kéna-cohen, Nat. Mater. 2014, 13, 271.

[10] J. D. Plumhof, T. Stoeferle, L. Mai, U. Scherf, R. F. Mahrt, Nat. Mater. 2014, 13, 328.

[11] N. G. Berloff, K. Kalinin, M. Silva, W. Langbein, P. G. Lagoudakis, ArXiv160706065 CondMat Physicsquant-Ph 2016.

[12] S. Kéna-cohen, S. R. Forrest, Nat. Photonics 2010, DOI 10.1038/nphoton.2010.86.

[13] C. P. Dietrich, A. Steude, L. Tropf, M. Schubert, N. M. Kronenberg, K. Ostermann, S. Höfling, M. C. Gather, Sci. Adv. 2016, 2, e1600666.

[14] N. Somaschi, L. Mouchliadis, D. Coles, I. E. Perakis, D. G. Lidzey, P. G. Lagoudakis, P. G. Savvidis, Appl. Phys. Lett. 2011, 99, 143303.

[15] R. T. Grant, P. Michetti, A. J. Musser, P. Gregoire, T. Virgili, E. Vella, M. Cavazzini, K. Georgiou, F. Galeotti, C. Clark, J. Clark, C. Silva, D. G. Lidzey, Adv. Opt. Mater. 2016.

[16] T. T. Vu, M. Dvorko, E. Y. Schmidt, J. F. Audibert, P. Retailleau, B. A. Trofimov, R. B. Pansu, G. Clavier, R. Méallet-Renault, J. Phys. Chem. C 2013.

[17] I. Mikhalyov, N. Gretskaya, F. Bergström, L. B.-A. Johansson, Phys. Chem. Chem. Phys. 2002, 4, 5663.

[18] S. Choi, J. Bouffard, Y. Kim, Chem. Sci. 2014, 5, 751.

[19] H. Deng, G. S. Solomon, R. Hey, K. H. Ploog, Y. Yamamoto, Phys. Rev. Lett. 2007, 99, 126403.

[20] G. Roumpos, M. Lohse, W. H. Nitsche, J. Keeling, M. H. Szymańska, P. B. Littlewood, A. Löffler, S. Höfling, L. Worschech, A. Forchel, Y. Yamamoto, Proc. Natl. Acad. Sci. 2012, 109, 6467.

[21] D. Caputo, D. Ballarini, G. Dagvadorj, C. S. Muñoz, M. De Giorgi, L. Dominici, K. West, L. N. Pfeiffer, G. Gigli, F. P. Laussy, M. H. Szymańska, D. Sanvitto, ArXiv161005737 Cond-Mat 2016.

[22] K. S. Daskalakis, S. A. Maier, S. Kéna-Cohen, Phys. Rev. Lett. 2015, 115, 35301. 


\section{Experimental methods}

\section{Sample preparation}

A polymer matrix solution was prepared using polystyrene (PS) having an average molecular weight $(\mathrm{Mw})$ of $\sim 192,000$ in toluene at a concentration of $35 \mathrm{mg} / \mathrm{ml}$. The PS/toluene solution was heated up to a temperature of $70^{\circ} \mathrm{C}$ and stirred for 30 minutes. BODIPY-Br was then added to the solution at a concentration of $10 \%$ by mass. Non-cavity films for absorption, photoluminescence and ASE measurements were spin-cast onto quartz-coated glass substrates.

For the microcavity fabrication a bottom 10-pair distributed Bragg reflector (DBR) of $\mathrm{SiO}_{2} / \mathrm{Nb}_{2} \mathrm{O}_{5}$ was deposited onto quartz-coated glass substrates using ion assisted electron beam $\left(\mathrm{Nb}_{2} \mathrm{O}_{5}\right)$ and thermal evaporation $\left(\mathrm{SiO}_{2}\right)$. The $186 \mathrm{~nm}$ thick BODIPY-Br active layer was then spin-coated on top of the bottom mirror. A second 8-pair DBR was deposited on top of the organic with the ion gun kept turned-off during the first few layers to avoid any damage on the organic material.

\section{Spectroscopy}

The absorption and PL measurements of the BODIPY-Br non-cavity films were performed using a Fluoromax 4 fluorometer (Horiba) that utilizes a Xe lamp. The angular white light reflectivity measurement was performed using a fibre-coupled Halogen-Deuterium white light source. A motorized arm was used to allow for the different illumination angles between the sample and the white light source. The reflected light was collected and coupled into an optical fibre mounted on a second motorized arm and then sent into a Andor Shamrock CCD spectrometer. 
Angular PL measurement were performed using the same motorized goniometer setup described earlier. A fibre-coupled Fianium supercontinuum laser with $6 \mathrm{ps}$ pulses and $40 \mathrm{MHz}$ repetition rates was used to non-resonantly excite the sample at 450nm using a bandpass filter. The sample was excited at a fixed angle of $45^{\circ}$ following a slight downwards tilt to the optical axis to avoid collection of the reflected excitation beam. An optical fibre on a motorized arm was used to collect a range of different angles with a resolution of $1^{\circ}$. The excitation density of the laser was kept relatively low $\left(1.2 \mu \mathrm{J} / \mathrm{cm}^{2}\right)$.

The ASE measurements were carried out using optical parametric amplifier (Coherent OPerA SOLO) pumped by high energy Ti:sapphire regenerative amplifier system (Coherent Libra-HE) providing up to $200 \mathrm{uJ}$ per pulse at $500 \mathrm{~nm}$, with $100 \mathrm{fs}$ pulse duration and $1 \mathrm{kHz}$ repetition rate. Line distributed beam was produced by Thorlabs ED1-L4100 line pattern diffuser and focused on the sample using $25.4 \mathrm{~mm}$ lens providing $0.173 \times 20 \mathrm{~mm}$ vertically polarized beam on the sample. Stimulated emission of BODIPY-Br thin film was detected from the edge of the film, in the direction of the strip and perpendicular to the propagation direction of the incident pump beam using Ocean Optics QE PRO spectrometer (0.7 nm spectral resolution). All measurements were performed at room temperature in air.

For the condensation study, the microcavity was excited non-resonantly at a wavelength of $400 \mathrm{~nm}$ using pulses at a $50 \mathrm{kHz}$ repetition rate and a pulse width $<2 \mathrm{ps}$ from a regenerative amplifier (Rega 9000 pumped with a Verdi V10, Coherent) seeded by a mode-locked picosecond Ti:Sapphire oscillator (Mira 900, coherent, pumped by the Millennia $X_{s}$, Spectra Physics) which was frequencydoubled through an optical parametric amplifier (OPA, Coherent). To prevent photooxidation of the sample, two optical choppers in a master-slave configuration with a modulation frequency of $28 \mathrm{~Hz}$ were used in conjunction with an optical shutter. This limited sample excitation to between 1 and 5 
pulses over a $30 \mathrm{~ms}$ exposure time. The sample was mounted in a vacuum chamber held under a dynamic vacuum of $10^{-6}$ mbar to further reduce photooxidation.

Photoluminescence was collected in transmission using an apochromatic Mitutoyo 50x microscope objective with a numerical aperture NA $=0.42$ and focused into a $550 \mathrm{~mm}$ spectrometer (Horiba Triax 550) coupled to an electron multiplying charged-coupled device (CCD) with a $500 \mathrm{~nm}$ longpass filter blocking the residual light from the excitation beam. The PL was spectrally and in-plane wavevector resolved using a 1200 grooves $/ \mathrm{mm}$ grating and a slit width of $100 \mu \mathrm{m}$ at the entrance of the spectrometer. The spatial coherence measurements were obtained by splitting the PL with a non-polarizing beam splitter in a Michelson interferometer configuration with the mirror on one arm replaced by a retroreflector. The PL was then coupled into the spectrometer with the grating at zero order to spatially resolve the PL on the EMCCD. 

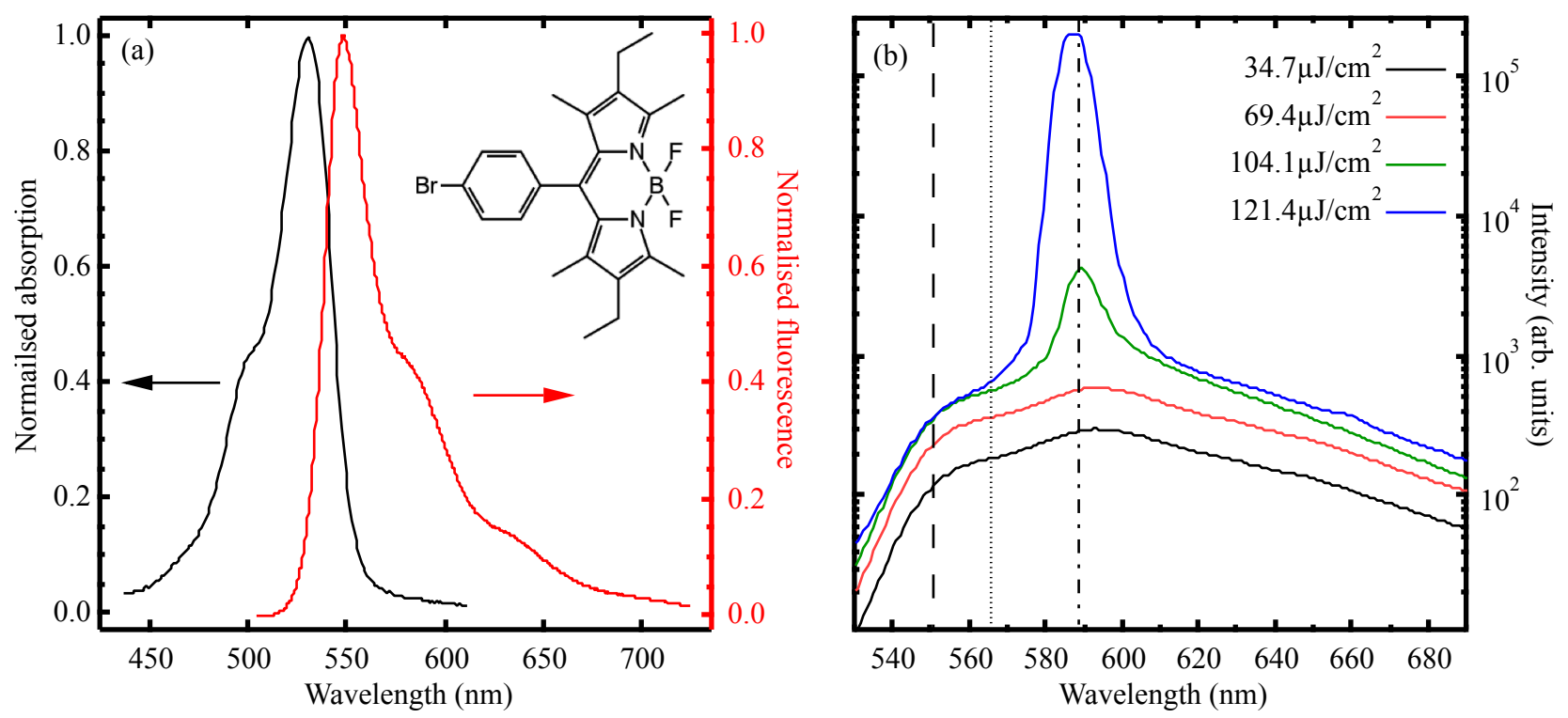

Figure 1 (a) The normalised absorption (black) and fluorescence spectrum (red) of BODIPY-Br dispersed in a transparent polystyrene matrix. The chemical structure is shown in the insert. (b) Amplified spontaneous emission from a 186nm thick film of the BODIPY-Br dispersed in a polystyrene matrix and deposited on a quartz substrate. A threshold is observed at a pump fluence of $104 \mu \mathrm{J} / \mathrm{cm}^{2}$ with a peak forming at $589 \mathrm{~nm}$ (dashed-dotted line). The dotted line indicates the polariton emission from the microcavity, whilst the large dashed line indicates the fluorscence emission peak shown in (a). 
(a)
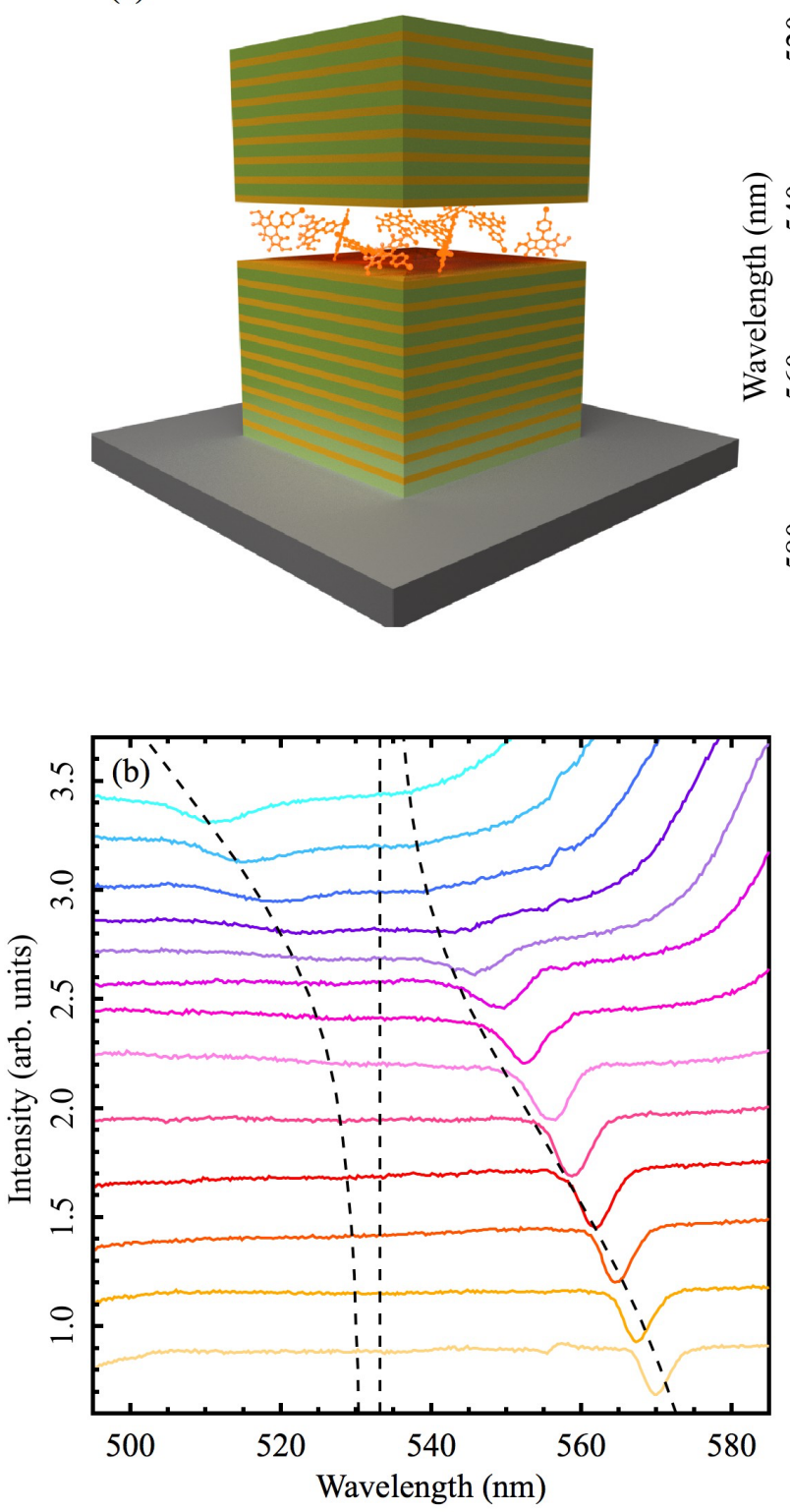
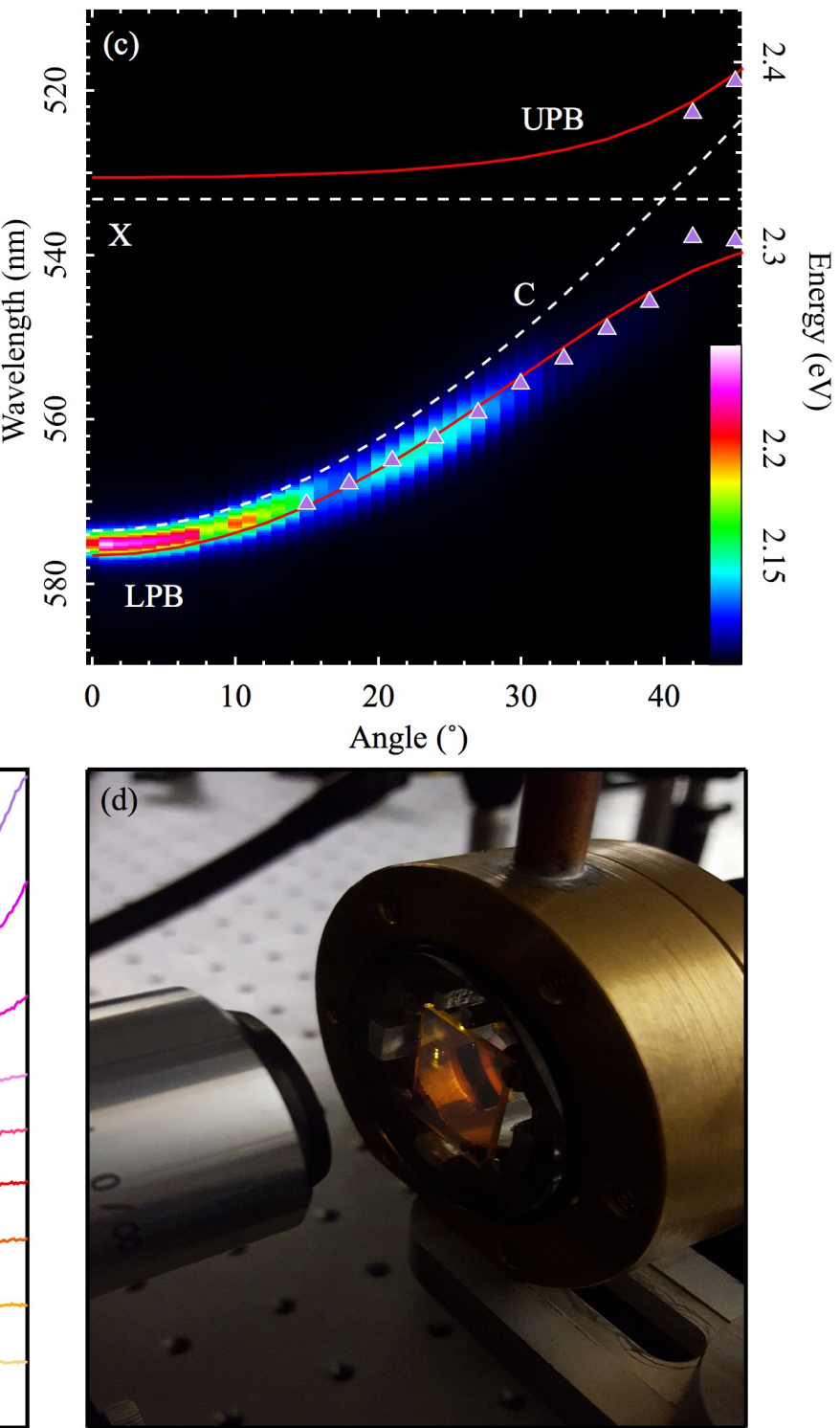

Figure 2 (a) Schematic of the dye filled microcavity. (b) Reflectivity spectra taken first at an angle of $15^{\circ}$ (light orange) and at every successive $3^{\circ}$ up to $51^{\circ}$ (light blue). The lower polariton branch is observed clearly in the smaller angles (orange to pink), whilst the anti-crossing can be observed from $42^{\circ}$ (dark purple) with the upper polariton branch becoming visible from that point onwards. The dashed lines indicate the upper polariton branch, exciton and lower polariton branch from left to right. (c) Polariton dispersion in the linear regime. The dispersion of the upper polariton (UPB) and lower polariton (LPB) branches are fitted using a standard two level oscillator model (red 
lines). The peaks from the reflectivity spectra in (b) are plotted as purple triangles showing a good fit to the polariton branches. (d) An image of the microcavity held in a vacuum chamber showing the yellow emission of the polariton condensate. 

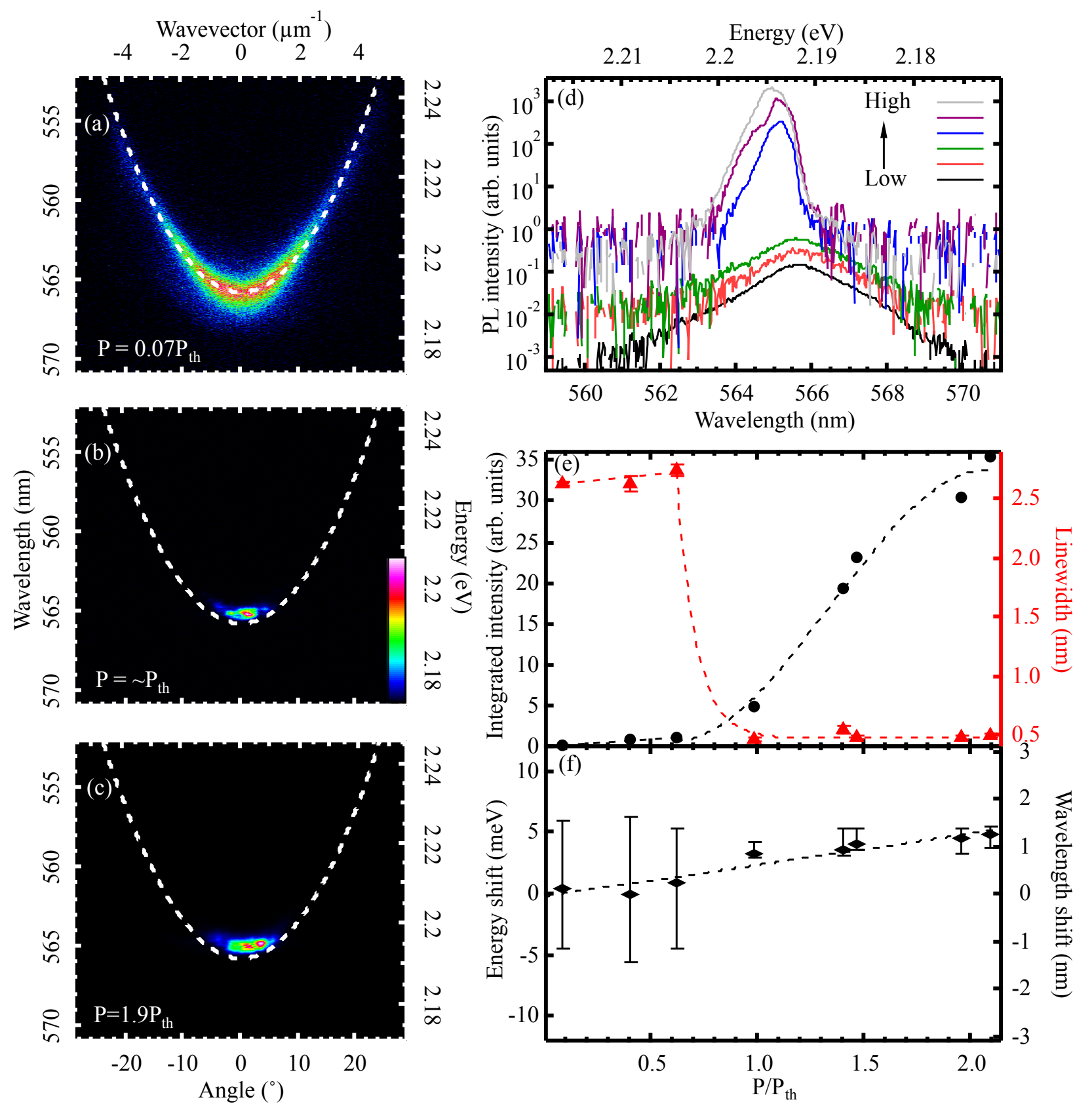

Figure 3 (a)-(c) Normalised dispersions taken below threshold, near threshold and above threshold. The white dashed line indicates the linear regime. (d) Photoluminescence spectra extracted at $\mathrm{k}_{/ /}=0$ from dispersion images recorded for different pump intensities and normalised to the number of excitation pulses used per recording. (e) Integrated intensity of the spectra and the corresponding linewidth measured at full width at half maximum (FWHM) as a function of threshold excitation density. (f) Energy shift of the photoluminescence spectrum as a function of threshold excitation density. 

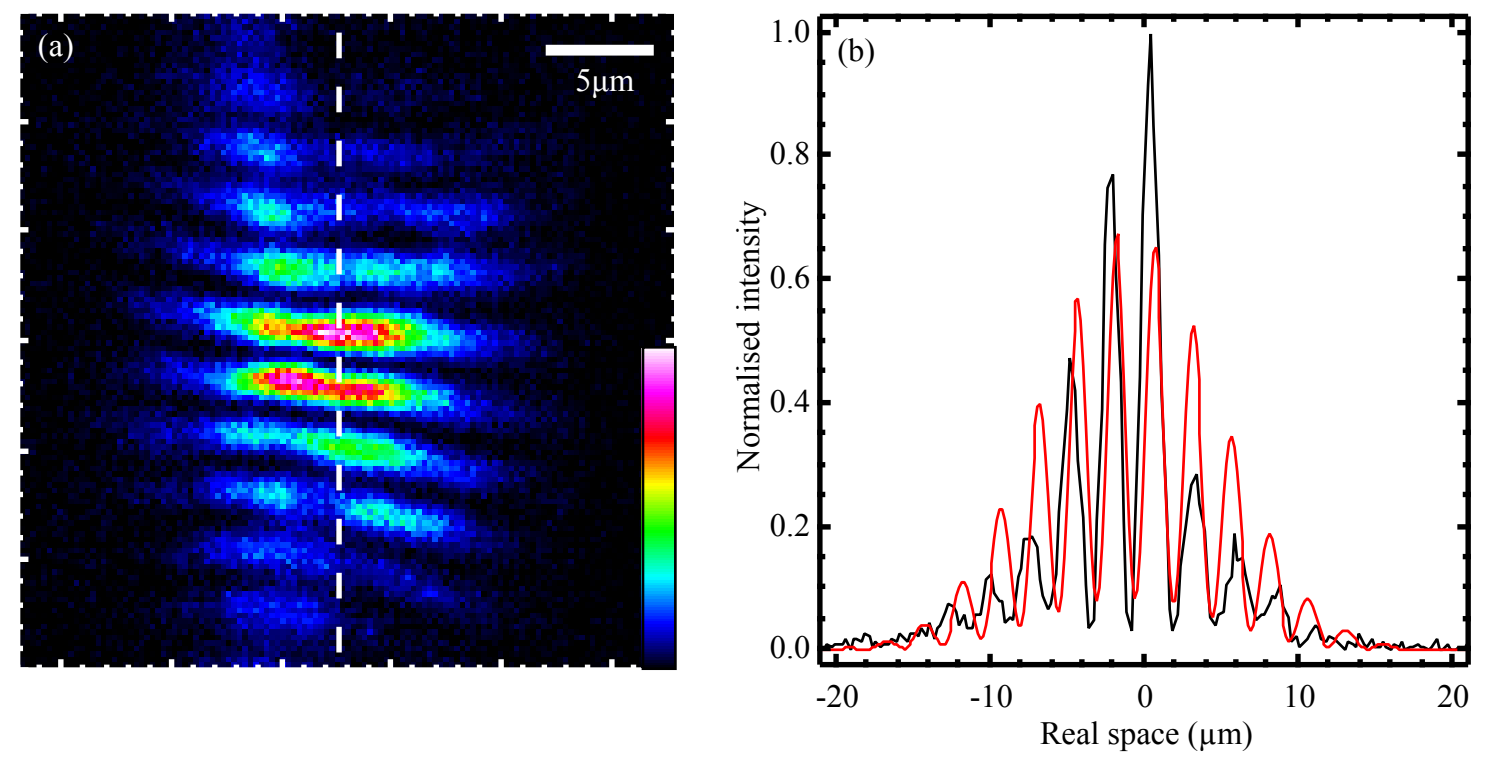

Figure 4 (a) Interferogram of a polariton condensate at threshold excitation density for a single excitation pulse. (b) Intensity profile (black line) taken along the white dashed line of (a) and the corresponding Gaussian fit to the data (red curve). 